\title{
Developing a Series Solution Method of $q$-Difference Equations
}

\author{
Hsuan-Ku Liu \\ Department of Mathematics and Information Education, National Taipei University of Education, Taiwan \\ Correspondence should be addressed to Hsuan-Ku Liu; hkliu.nccu@gmail.com
}

Received 16 November 2012; Accepted 14 April 2013

Academic Editor: Filomena Cianciaruso

Copyright (c) 2013 Hsuan-Ku Liu. This is an open access article distributed under the Creative Commons Attribution License, which permits unrestricted use, distribution, and reproduction in any medium, provided the original work is properly cited.

The series solution is widely applied to differential equations on $\mathbb{R}$ but is not found in $q$-differential equations. Applying the Taylor and multiplication rule of two generalized polynomials, we develop a series solution of linear homogeneous $q$-difference equations. As an example, the series solution method is used to find a series solution of the second-order $q$-difference equation of Hermite's type.

\section{Introduction}

Several works have been done recently for series solutions on certain time scales. One of the difficulties for developing a theory of series solutions for linear homogeneous equations on time scales is that the formula for multiplication by two generalized polynomials is not easily found. If the time scale has constant graininess, Haile and Hall [1] provided an exact formula for the multiplicity of two generalized polynomials. Using the obtained results, the series solutions for linear dynamic equations are proposed on the time scales $\mathbb{R}$ and $\mathbb{T}=$ $h \mathbb{Z}$ (difference equations with step size $h$ ). On generalized time scales, Mozyrska and Pawłuszewicz [2] presented the formula for the multiplicity of the generalized polynomials of degree one and degree $n \in \mathbb{N}$.

Let $0<q<1$ and use the notations

$$
q^{\mathbb{N}}=\left\{q^{n} \mid n \in \mathbb{N}\right\}, \quad \overline{q^{\mathbb{N}}}=q^{\mathbb{N}} \cup\{0\},
$$

where $\mathbb{N}$ denotes the set of positive integers. Liu [3] presented a formula for the multiplication of two $q$-polynomials. The obtained results are used to develop a series solution method of the second-order difference equations on $\overline{q^{\mathbb{N}}}$. Precisely, the second-order $q$-difference equation is described as

$$
u^{\Delta \Delta}(t)+g(t) u^{\Delta}(t)+f(t) u(t)=0, \quad t \in \overline{q^{\mathbb{N}}}
$$

where $f$ and $g$ are both $q$-analytic functions at 0 in the interval $(c, d)$. As an example, the series solution method is applied to consider the $q$-Hermite's equation of the form

$$
u^{\Delta \Delta}(t)-t u^{\Delta}(t)+\lambda u(t)=0, \quad t \in \overline{q^{\mathbb{N}}}
$$

with initial condition $u(0)=a$ and $u^{\Delta}(0)=b$.

This paper is organized as follows: in Section 2, basic ideas on $q$-calculus are introduced. The series solution method is developed in Section 3 and is applied to consider the $q$ Hermite's equation in Section 4. Finally, a concise conclusion is provided in Section 5.

\section{A Basic Introduction to Time Scales}

A time scale means an arbitrary nonempty closed subset of the real numbers. The calculus of time scales was initiated by Liu [3] in order to create a theory that can unify discrete and continuous analysis.

Then, we introduce the delta derivative by starting to define the forward and backward jump operators.

Definition 1. Let $\mathbb{T}$ be a time scale. For $t \in \mathbb{T}$, we define the forward jump operator $\sigma: \mathbb{T} \rightarrow \mathbb{T}$ by

$$
\sigma(t):=\inf \{s>t \mid s \in \mathbb{T}\},
$$

while the backward jump operator $\rho: \mathbb{T} \rightarrow \mathbb{T}$ by

$$
\rho(t):=\sup \{s<t \mid s \in \mathbb{T}\} .
$$


Definition 2. The graininess function $\mu: \mathbb{T} \rightarrow[0, \infty)$ is defined by

$$
\mu(t):=\sigma(t)-t
$$

According to the basic definitions, we can give some useful relationships concerning the delta derivative.

Let $a$ and $q$ be real numbers such that $0<q<1$. The $q$-shift factorial [4] is defined by

$$
(a ; q)_{0}=1, \quad(a ; q)_{n}=\prod_{k=0}^{n-1}\left(1-a q^{k}\right), \quad n=1,2, \ldots, n .
$$

Assume $f: \mathbb{T} \rightarrow \mathbb{R}$ is a function and $t \in \mathbb{T}$. The $q$ derivative [5] at $t$ is defined by

$$
f^{\Delta}(t)=\frac{f(q t)-f(t)}{(q-1) t} .
$$

A $q$-difference equation is an equation that contains $q$ derivatives of a function defined on $\overline{q^{\mathbb{N}}}$.

Definition 3. On the time scale $\mathbb{T}$, the $q$-polynomials $h_{k}\left(\cdot, t_{0}\right)$ : $\mathbb{} \mathbb{R}$ are defined recursively as follows:

$$
h_{0}(t, s)=1, \quad h_{k+1}=\int_{s}^{t} h_{k}(\tau, s) \Delta \tau \text {. }
$$

Hence, for each fixed $s$, the delta derivative of $h_{k}$ with respect to $t$ satisfies

$$
h_{k}^{\Delta}(t, s)=h_{k-1}(t, s), \quad k \geq 1 .
$$

By computing the recurrence relation, the $q$-polynomials can be represented as

$$
h_{k}(t, s)=\prod_{\nu=0}^{k-1} \frac{t-s q^{\nu}}{\sum_{j=0}^{v} q^{j}}
$$

on $\overline{q^{\mathbb{N}}}[5]$.

Agarwal and Bohner [6] give a Taylor's formula for functions on a general time scale. On $\overline{q^{\mathbb{N}}}$, the Taylor's formula can be rewritten as the following form.

Theorem 4. Let $n \in \mathbb{N}$. Suppose $f$ is $n$ times differentiable on $\overline{q^{\mathbb{N}}}$. Let $\alpha, t \in \overline{q^{\mathbb{N}}}$. One has

$$
f(t)=\sum_{k=0}^{n-1} h_{k} f^{\Delta^{k}}(\alpha)+\int_{\alpha}^{\rho^{n-1}(t)} h_{n-1}(t, \sigma(\tau)) f^{\Delta^{n}}(\tau) \Delta \tau .
$$

Before developing the series solution method, we introduce the $q$-analytic function on $\overline{q^{\mathbb{N}}}$.

Definition 5. A real-valued function $f: \overline{q^{\mathbb{N}}} \rightarrow \mathbb{R}$ is said to be $q$-analytic at $t_{0}$ if and only if there is a power series centered at $t_{0}$ that converges to $f$ near $t_{0}$; that is, there exist coefficients $\left\{a_{k}\right\}_{k=0}^{\infty}$ and points $c, d \in \overline{q^{\mathbb{N}}}$ such that $c<t_{0}<d$ and

$$
f(t)=\sum_{k=0}^{\infty} a_{k} h_{k}\left(t, t_{0}\right)
$$

for all $t \in(c, d) \cap \overline{q^{\mathbb{N}}}$.

The production rule of two $q$-polynomials at 0 which will be used to derive the series solution in following sections [3].

Theorem 6. Let $h_{i}(t, 0)$ and $h_{j}(t, 0)$ be two q-polynomials at zero. One has

$$
h_{i}(t, 0) h_{j}(t, 0)=\frac{\left(q^{i+1} ; q\right)_{j}}{(q ; q)_{j}} h_{i+j}(t, 0) .
$$

Proof. Since

$$
h_{i+j}(t, 0)=\prod_{\nu=0}^{i+j-1} \frac{t}{\sum_{\mu=0}^{\nu} q^{\mu}},
$$

we have

$h_{i+j}(t, 0)$

$$
\begin{aligned}
& =\left(\prod_{\nu=0}^{i-1} \frac{t}{\sum_{\mu=0}^{\nu} q^{\mu}}\right)\left(\prod_{\nu=i}^{i+j-1} \frac{t}{\sum_{\mu=0}^{\nu} q^{\mu}}\right) \\
& =h_{i}(t, 0)\left(\frac{\prod_{\nu=0}^{j-1} \sum_{\mu=0}^{\nu} q^{\mu}}{\prod_{\nu=0}^{j-1} \sum_{\mu=0}^{\nu} q^{\mu}}\right) t^{j}\left(\prod_{\nu=i}^{i+j-1} \frac{1}{\sum_{\mu=0}^{\nu} q^{\mu}}\right) \\
& =h_{i}(t, 0)\left(\prod_{\nu=0}^{j-1} \frac{t}{\sum_{\mu=0}^{\nu} q^{\mu}}\right)\left(\prod_{\nu=0}^{j-1} \sum_{\mu=0}^{\nu} q^{\mu}\right)\left(\prod_{\nu=i}^{i+j-1} \frac{1}{\sum_{\mu=0}^{\nu} q^{\mu}}\right) \\
& =h_{i}(t, 0) h_{j}(t, 0)\left(\prod_{\nu=0}^{j-1} \frac{\sum_{\mu=0}^{\nu} q^{\mu}}{\sum_{\mu=0}^{\nu+i} q^{\mu}}\right) .
\end{aligned}
$$

This implies that

$$
\begin{aligned}
h_{i}(t, 0) h_{j}(t, 0) & =\left(\prod_{\nu=0}^{j-1} \frac{\sum_{\mu=0}^{\nu+i} q^{\mu}}{\sum_{\mu=0}^{\nu} q^{\mu}}\right) h_{i+j}(t, 0) \\
& =\prod_{\nu=0}^{j-1} \frac{\left(1-q^{v+i+1}\right)}{\left(1-q^{v+1}\right)} h_{i+j}(t, 0) \\
& =\frac{\left(q^{i+1} ; q\right)_{j}}{(q ; q)_{j}} h_{i+j}(t, 0) .
\end{aligned}
$$

Proposition 7. Let $h_{i}(t)$ and $h_{j}(t)$ be any two q-polynomials. One has

$$
h_{i}(t, 0) h_{j}(t, 0)=h_{j}(t, 0) h_{i}(t, 0) \text {. }
$$


Proof. Without loss of generality, we suppose $i>j$ and have

$$
\begin{aligned}
& \frac{\left(q^{i+1} ; q\right)_{j}}{(q, q)_{j}}-\frac{\left(q^{j+1} ; q\right)_{i}}{(q, q)_{i}} \\
& =\frac{\left(1-q^{j+1}\right) \cdots\left(1-q^{i+j}\right)}{(1-q) \cdots\left(1-q^{i}\right)}-\frac{\left(1-q^{i+1}\right) \cdots\left(1-q^{i+j}\right)}{(1-q) \cdots\left(1-q^{j}\right)} \\
& =\frac{\left(1-q^{j+1}\right) \cdots\left(1-q^{i+j}\right)}{(1-q) \cdots\left(1-q^{i}\right)} \\
& \quad-\frac{\left(1-q^{i+1}\right) \cdots\left(1-q^{i+j}\right)\left(1-q^{j+1}\right) \cdots\left(1-q^{i}\right)}{(1-q) \cdots\left(1-q^{j}\right)\left(1-q^{j+1}\right) \cdots\left(1-q^{i}\right)} \\
& =0 .
\end{aligned}
$$

This implies that

$$
\frac{\left(q^{i+1} ; q\right)_{j}}{(q, q)_{j}}=\frac{\left(q^{j+1} ; q\right)_{i}}{(q, q)_{i}}
$$

Therefore, we have

$$
h_{i}(t, 0) h_{j}(t, 0)=h_{j}(t, 0) h_{i}(t, 0)
$$

by Theorem 6 .

\section{Developing Series Solutions Method}

Using the Taylor series on time scales, we develop a series solution method for solving $q$-difference equations in this section.

Consider a second-order $q$-difference equation

$$
u^{\Delta \Delta}(t)+g(t) u^{\Delta}(t)+f(t) u(t)=0, \quad t \in \overline{q^{\mathbb{N}}},
$$

where $f$ and $g$ are both $q$-analytic functions at 0 in the interval $(c, d)$. Hence, there exist two sequences of coefficients $\{F(k)\}$ and $\{G(k)\}$ such that

$$
f(t)=\sum_{k=0}^{\infty} F(k) h_{k}(t, 0), \quad g(t)=\sum_{k=0}^{\infty} G(k) h_{k}(t, 0)
$$

for all $t \in(c, d) \cap \overline{q^{\mathbb{N}}}$.

One can find a power series solution of the form

$$
u(t)=\sum_{k=0}^{\infty} U(k) h_{k}(t, 0),
$$

by carrying out the following steps.

Step 1. Since

$$
\begin{aligned}
& u^{\Delta}(t)=\sum_{k=0}^{\infty} U(k+1) h_{k}(t, 0), \\
& u^{\Delta \Delta}(t)=\sum_{k=0}^{\infty} U(k+2) h_{k}(t, 0),
\end{aligned}
$$

we get

$$
\begin{aligned}
g(t) & u^{\Delta}(t) \\
& =\left[\sum_{k=0}^{\infty} G(k) h_{k}(t, 0)\right]\left[\sum_{l=0}^{\infty} U(l+1) h_{l}(t, 0)\right] \\
& =\sum_{k=0}^{\infty} \sum_{l=0}^{k} G(l) U(k+1-l)\left(h_{l}(t, 0) h_{k-l}(t, 0)\right) \\
& =\sum_{k=0}^{\infty}\left[\sum_{l=0}^{k} G(l) U(k+1-l) \frac{\left(q^{l+1}, q\right)_{k-l}}{(q, q)_{k-l}}\right] h_{k}(t, 0), \\
f(t) & u(t) \\
= & {\left[\sum_{k=0}^{\infty} F(k) h_{k}(t, 0)\right]\left[\sum_{l=0}^{\infty} U(l) h_{l}(t, 0)\right] } \\
= & \sum_{k=0}^{\infty} \sum_{l=0}^{k} F(l) U(k-l)\left(h_{l}(t, 0) h_{k-l}(t, 0)\right) \\
= & \sum_{k=0}^{\infty}\left[\sum_{l=0}^{k} F(l) U(k-l) \frac{\left(q^{l+1}, q\right)_{k-l}}{(q, q)_{k-l}}\right] h_{k}(t, 0) .
\end{aligned}
$$

Substituting (24) and (26) into (22), we get the equation

$$
\sum_{k=0}^{\infty}\left[\begin{array}{l}
U(k+2) \\
+\sum_{l=0}^{k} G(l) U(k+1-l) \frac{\left(q^{l+1}, q\right)_{k-l}}{(q, q)_{k-l}} \\
+\sum_{l=0}^{k} F(l) U(k-l) \frac{\left(q^{l+1}, q\right)_{k-l}}{(q, q)_{k-l}}
\end{array}\right] h_{k}(t, 0)=0
$$

Step 2. Set the coefficients of the power series equal to zero. That gives a recurrence relation that relates later coefficients in the power series (24) to the earlier ones. That is,

$$
\begin{gathered}
U(k+2)+\sum_{l=0}^{k} G(l) U(k+1-l) \frac{\left(q^{l+1}, q\right)_{k-l}}{(q, q)_{k-l}} \\
+\sum_{l=0}^{k} F(l) U(k-l) \frac{\left(q^{l+1}, q\right)_{k-l}}{(q, q)_{k-l}}=0 .
\end{gathered}
$$

Step 3. Find all coefficients $U(k)$ in terms of the first two coefficients $U(0)$ and $U(1)$, thus writing the $q$-series in the form

$$
\sum_{k=0}^{\infty} U(k) h_{k}(t, 0)=U(0) u_{1}(t)+U(1) u_{2}(t)
$$

where $u_{1}$ and $u_{2}$ are two linearly independent $q$-series solutions. 


\section{Applications}

In this section, the series solution method is applied to consider the q-Hermite's equations with initial conditions.

Consider the $q$-Hermite's equation of the form

$$
u^{\Delta \Delta}(t)-t u^{\Delta}(t)+\lambda u(t)=0, \quad t \in \overline{q^{\mathbb{N}}}
$$

with $u(0)=a$ and $u^{\Delta}(0)=b$.

Let

$$
u(t)=\sum_{k=0}^{\infty} U(k) h_{k}(t, 0)
$$

then $u(0)=a=U(0)$ and $u^{\Delta}(0)=b=U(1)$. Applying (31) into (30), we have

$$
\begin{gathered}
U(2)=-\lambda U(0), \\
U(k+2)=\left[\frac{\left(q^{k} ; q\right)_{1}}{(q ; q)_{1}}-\lambda\right] U(k)=\left[\frac{\left(1-q^{k}\right)}{(1-q)}-\lambda\right] U(k),
\end{gathered}
$$

where $k=1,2, \ldots$.

This implies that

$$
\begin{aligned}
U(2 l) & =\left[\frac{\left(1-q^{2(l-1)}\right)}{(1-q)}-\lambda\right] U(2(l-1)) \\
& =\prod_{i=1}^{l}\left[\frac{\left(1-q^{2(i-1)}\right)}{(1-q)}-\lambda\right] a, \\
U(2 l+1) & =\left[\frac{\left(1-q^{2 l-1}\right)}{(1-q)}-\lambda\right] U(2 l-1) \\
& =\prod_{i=1}^{l}\left[\frac{\left(1-q^{2 i-1}\right)}{(1-q)}-\lambda\right] b .
\end{aligned}
$$

Hence, we get

$$
\begin{aligned}
u(t)= & a\left(1+\sum_{l=1}^{\infty} \prod_{i=1}^{l}\left[\frac{\left(1-q^{2(i-1)}\right)}{(1-q)}-\lambda\right] h_{2 l}(t)\right) \\
& +b\left(h_{1}(t)+\sum_{l=1}^{\infty} \prod_{i=1}^{l}\left[\frac{\left(1-q^{2 i-1}\right)}{(1-q)}-\lambda\right] h_{2 l+1}(t)\right) \\
= & a u_{1}(t)+b u_{2}(t) .
\end{aligned}
$$

By computing the Wronskian of $u_{1}$ and $u_{2}$ at 0 , we get

$$
W\left[u_{1}, u_{2}\right](0)=u_{1}(0) u_{2}^{\Delta}(0)-u_{2}(0) u_{1}^{\Delta}(0)=1 \neq 0 .
$$

This implies that $u_{1}$ and $u_{2}$ are two linearly independent solutions.
Example 8. Consider the $q$-Hermite's equation with $q=1 / 2$ of the form

$$
u^{\Delta \Delta}-t u^{\Delta}+u=0
$$

with $u(0)=1$ and $u^{\Delta}=0$. Substituting (31) into (36) yields

$$
U(2 l)=\Pi_{i=1}^{l}\left[\frac{1-(1 / 2)^{2(i-1)}}{(1 / 2)}-1\right]=\Pi_{i=1}^{l}\left[1-\left(\frac{1}{2}\right)^{2 i-3}\right]
$$

and $U(2 l+1)=0$ which implies that

$$
\begin{aligned}
u(t) & =1+\sum_{l=1}^{\infty} \Pi_{i=1}^{l} 1-\left(\frac{1}{2}\right)^{2 i-3} h_{2 l}(t) \\
& =1-h_{2}(t)-\frac{1}{2} h_{4}(t)-\frac{3}{8} h_{6}(t)-\cdots .
\end{aligned}
$$

\section{Conclusion}

One area which is the lack of development is the theory of series solutions on $q$-difference equations. In this paper, we present the formula for the multiplicity of two $q$-polynomials at 0 . The purpose is to provide the basic mechanics for finding the series solutions of linear homogeneous $q$-difference equation. As an example we consider series solution of the $q$ Hermite's equation of the form $u^{\Delta \Delta}-t u^{\Delta}+\lambda u=0$, with the initial conditions $u(0)=a$ and $u^{\Delta}(0)=b$. Using the presented method, the series solution of the Hermite's equation can be obtained iteratively. In future studies, we would apply the presented method to find the series solution of other $q$ difference equations on $\overline{q^{\mathbb{N}}}$.

\section{References}

[1] B. D. Haile and L. M. Hall, "Polynomial and series solutions of dynamic equations on time scales," Dynamic Systems and Applications, vol. 12, no. 1-2, pp. 149-157, 2003.

[2] D. Mozyrska and E. Pawłuszewicz, "Hermite’s equations on time scales," Applied Mathematics Letters, vol. 22, no. 8, pp. 1217-1219, 2009.

[3] H.-K. Liu, "Application of a differential transformation method to strongly nonlinear damped q-difference equations," Computers \& Mathematics with Applications, vol. 61, no. 9, pp. 25552561, 2011.

[4] T. H. Koornwinder, " $q$-special functions: a tutorial," http://arxiv.org/abs/math/9403216.

[5] M. Bohner and A. Peterson, Dynamic Equations on Time Scales: An Introduction with Applications, Birkhäuser, Boston, Mass, USA, 2001.

[6] R. P. Agarwal and M. Bohner, "Basic calculus on time scales and some of its applications," Results in Mathematics, vol. 35, no. 1-2, pp. 3-22, 1999. 


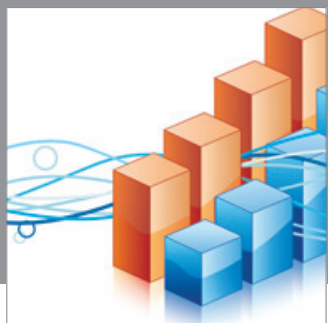

Advances in

Operations Research

mansans

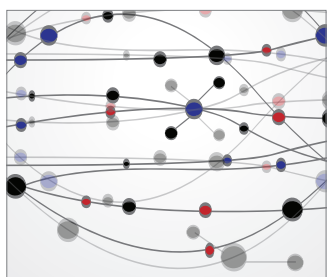

The Scientific World Journal
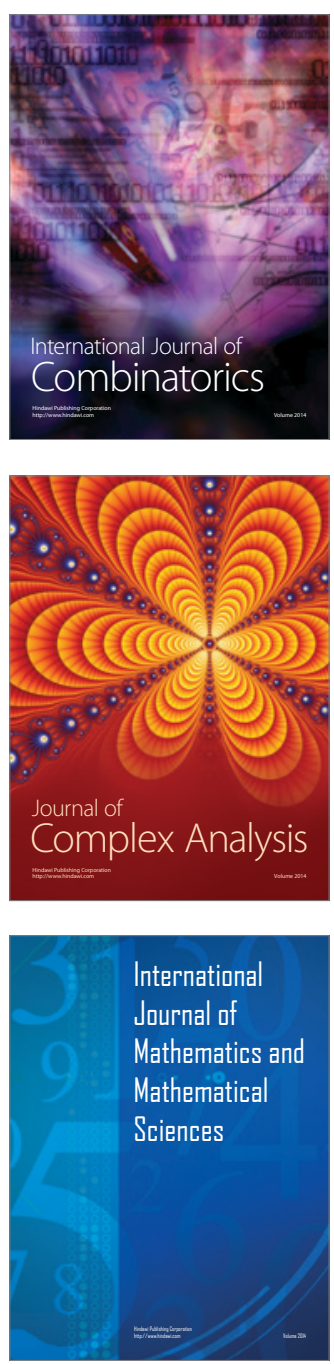
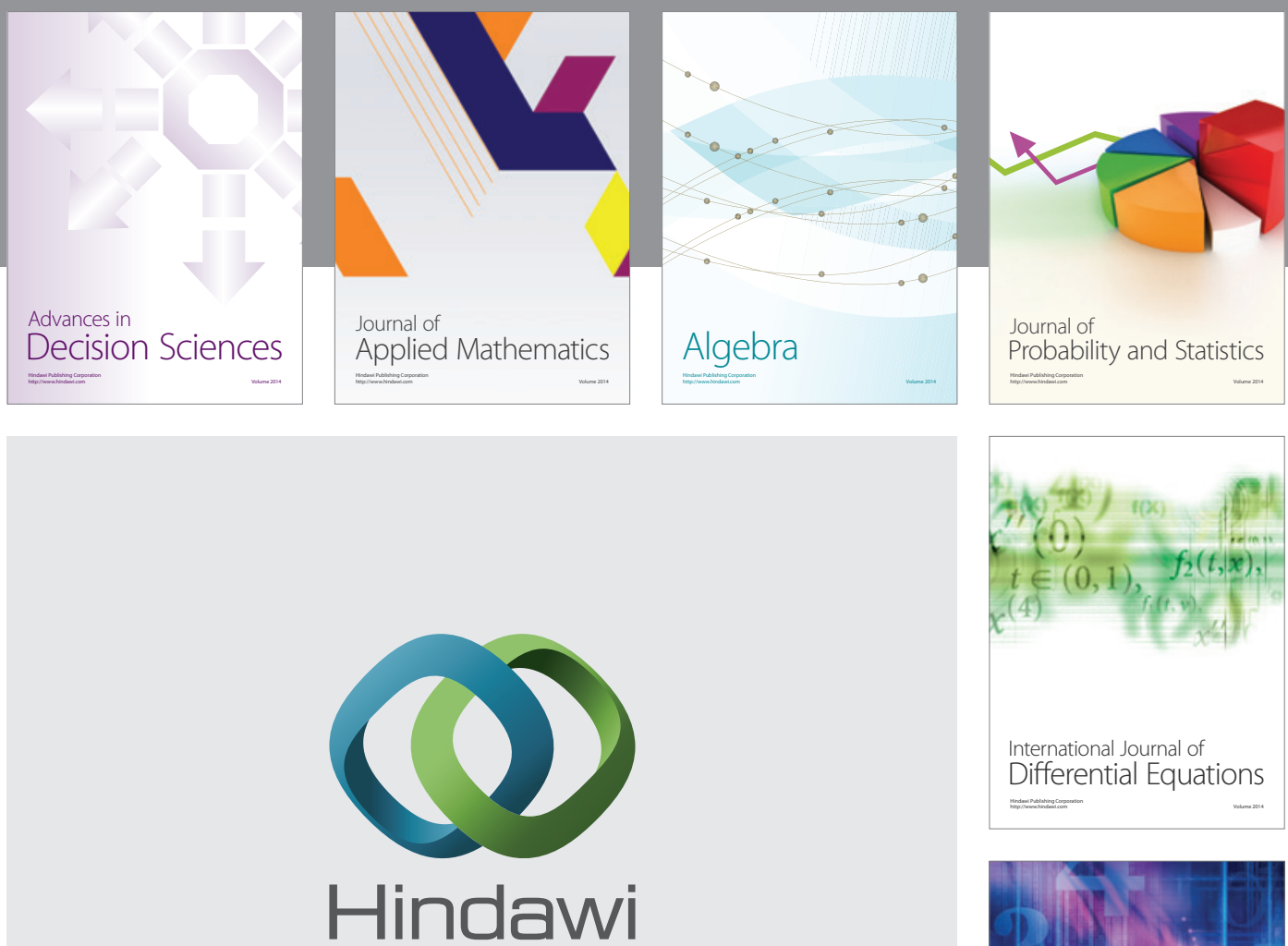

Submit your manuscripts at http://www.hindawi.com
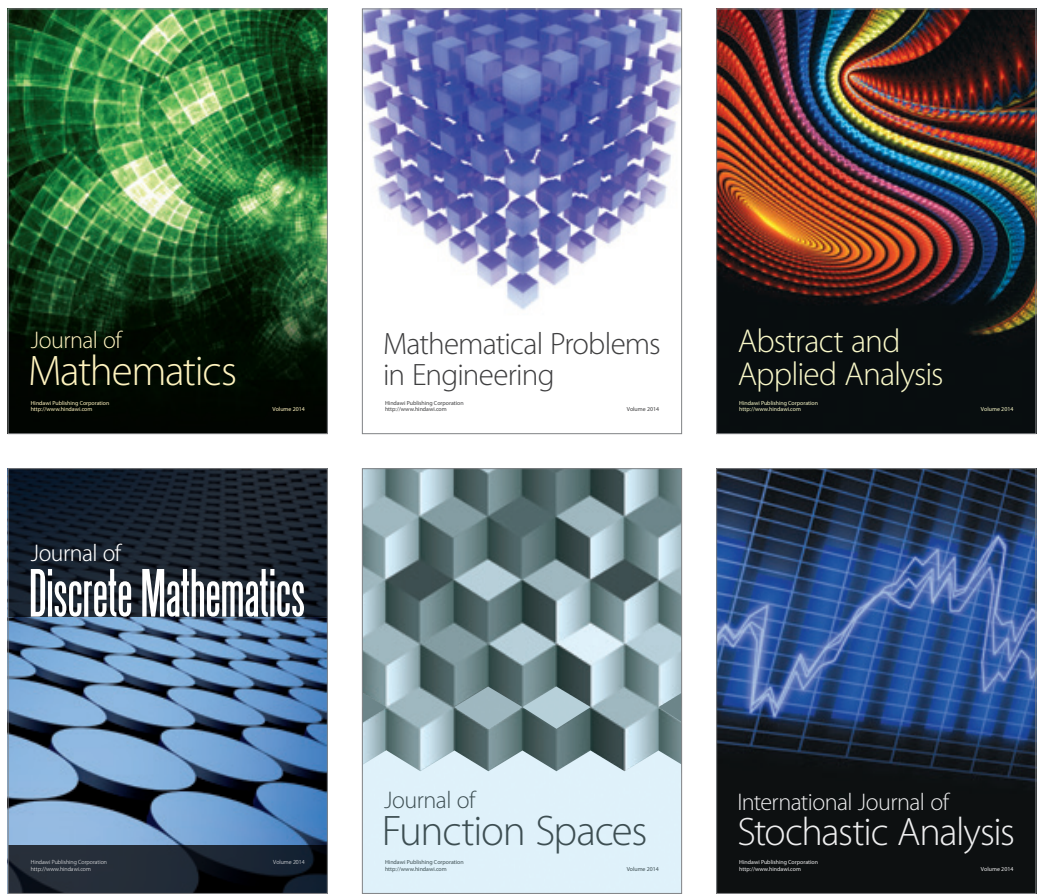

Journal of

Function Spaces

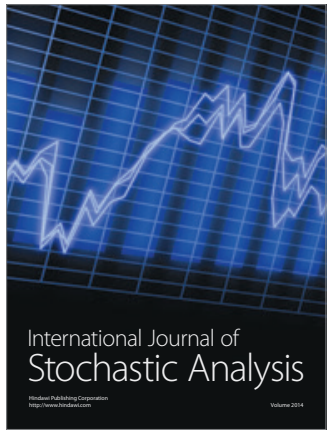

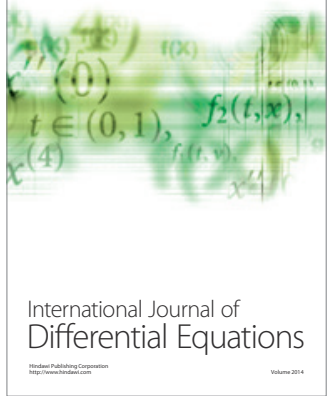
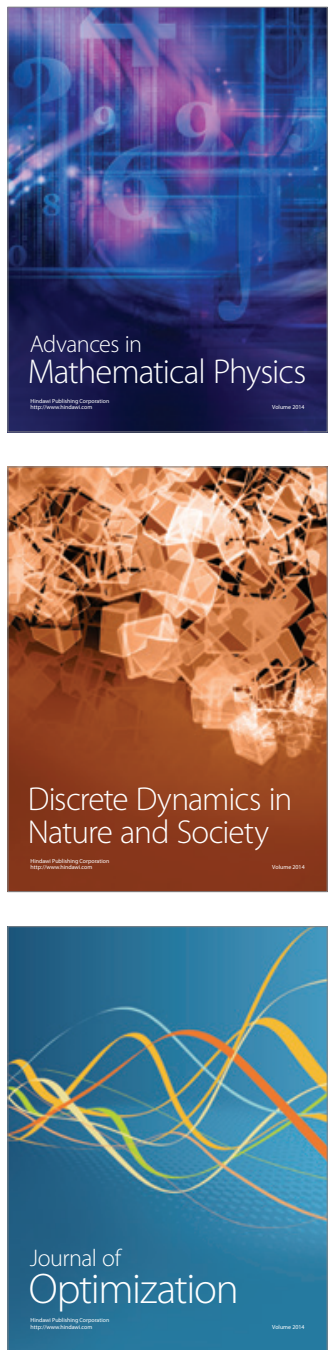